\title{
Role of Cardiac Magnetic Resonance Imaging in Improving Prognostic Assessment of Adverse Cardiac Events in Dilated Cardiomyopathies
}

\author{
Alberto Roghi*, Stefano Pedretti, Matteo Baroni, Paola Sormani, Angela Milazzo, Giuseppina Quattrocchi, \\ and Patrizia Pedrotti
}

CMR Unit, Department of Cardiology and Cardiac Surgery, Niguarda ASST Grande Ospedale Metropolitano, Milan, Italy

Received: February 02, 2018; Published: February 08, 2018

*Corresponding author: Alberto Roghi, CMR Unit, Department of Cardiology and Cardiac Surgery, Niguarda ASST Grande Ospedale Metropolitano, Milan, Italy, Email: alberto.roghi@gmail.com

Abbreviations: CMR: Cardiac Magnetic Resonance; SCD: Sudden Cardiac Death; VA: Ventricular Arrhythmias; DCM: Dilated Cardiomyopathy; ICD: Cardioverter Defibrillator; LVEF: Left Ventricular Ejection Fraction

\section{Opinion}

Cardiac Magnetic Resonance (CMR) has a relevant role in the comprehensive assessment of cardiac function, morphology and myocardial characterization.The high spatial resolution offers precise evaluation of cardiac volumes without geometric assumptions and myocardial characterization of edema, fat infiltration and fibrosis offers a sort of "in-vivo" virtual pathology. For these reasons the role of CMR imaging in the diagnostic workup of cardiomyopathies has gained increasing relevance, improving the assessment of many relevant issues as the etiology of sudden cardiac arrest [1], the evidence and extension of myocardial fibrosis in ischemic and non-ischemic cardiomyopathies [2,3], the evidence of fat and fibrotic infiltration in arrhytmogenic cardiomyopathies $[4,5]$. The relatively scarce literature supporting a prognostic value of CMR imaging data is due to the short history of MR imaging in cardiology. Recently, an increasing number of reports is supporting the role of CMR data in the prognostic assessment of adverse cardiac events in dilated cardiomyopathies, raising relevant clinical and financial issues in the management of patients.

Sudden Cardiac Death (SCD) and malignant ventricular arrhythmias (VA) constitute a major cause of death in patients affected by dilated cardiomyopathy (DCM) of various etiologies. The Implantable Cardioverter Defibrillator (ICD) has been shown to be effective in detecting and treating such arrhythmias. Indeed, in various clinical settings, ICD patients have displayed marked reductions in mortality [6,7]. However, SCD risk stratification is still suboptimal, particularly in primary prevention. In a MADIT II post-hoc analysis, only $35 \%$ of the patients who received an ICD required antiarrhythmic therapy during a 3-year follow-up [7] and in multicenter randomised trials [8] the number of implants needed to prevent a major arrhythmic event has been seen to range from 3 to 11 . In this category, candidacy for primary prevention ICD implantation is based essentially on etiology, left ventricular ejection fraction (LVEF) and NYHA functional class [9].

Concerning DCM of nonischemic etiology (NIDCM), the recently published results from DANISH trial show that ICD implantation for primary prevention based solely on LVEF may not result in a clinical benefit on survival [10]. LVEF is a marker of systolic dysfunction, easily obtained by 2D-echocardiography and largely diffused for diagnosis and follow-up. However, the assessment of LVEF by 2D echocardiography shows two main limitations:

i. The intrinsic limitation of the ejection fraction, which is a trivial ratio between systolic output and end-diastolic volume independent from ventricle dilatation.

ii. The methodological limitations related to the poor spatial resolution and geometric assumption to assess right and left ventricular volumes. Although echo-LVEF has been for a long time the most powerful prognostic predictor in cardiomyopathies, a more precise indicator for those patients with more advance disease is warranted in order to tailor their management, improving both treatment and cost effectiveness. In the end-stage disease phase of cardiomyopathy the depressed LVEF is per se a poor prognostic indicator whilst the assessment of cardiac dilatation by precise evaluation of left and right volumes, as well as the recognition of concomitant mitral regurgitation is more effective for improving the prognostic 
assessment. In a recent revision of a cohort of 96 patients with ischemic and non-ischemic DCM who received ICD, the most important predictors of major adverse cardiac events (death, heart transplantation, ventricular mechanical device) during a 76 month follow-up were LV end-diastolic volume and RV dysfunction, while the most important predictor of VA and ICD intervention was LGE assessed by CMR [11]. Several studies have shown that myocardial scarring, fibrosis and bordering transition tissue make up most of the arrhythmogenic substrate in ischemic cardiomyopathy (IDCM) and in more than $80 \%$ of NIDCM [12-13]. CMR by means of late gadolinium enhancement (LGE) imaging is currently considered the reference technique for detecting, quantifying and describing myocardial fibrosis, and displays high precision and reproducibility. Concerning NIDCM, the majority of researches considered the presence of LGE as a potential marker of adverse clinical and arrhythmic outcome. Recent literature data confirm the presence of midwall LGE as a negative prognostic marker for patients with DCM [14].

\section{Conclusion}

In conclusion, there is increasing evidence that CMR morphologic, functional and tissue characterization data improve the prognostic assessment of adverse cardiac events in ischemic and nonischemic cardiomyopathies with incremental value on clinical and echocardiographic predictors. An accurate assessment of right and left ventricular volumes gives important information on cardiac dilatation with significant correlations with adverse prognosis, even in patients with end-stage disease with depressed LVEF. Pattern and extension of myocardial fibrosis identify patients at highest risk of arrhythmias who benefit from ICD implantation.

\section{References}

1. White JA, Fine NM, Gula L, YeeR, Skanes A, et al. (2012) Utility of Cardiac Magnetic Resonance in Identifying Substrate for Malignant Ventricular Arrhythmias. Circulation: Cardiovascular Imaging 5:12-20.

2. Mahrholdt H, Wagner A, Holly TA, Elliott MD, Bonow RO, et al. (2002) Reproducibility of chronic infarct size measurement by contrastenhanced magnetic resonance imaging. Circulation 106(18):2322-2327.

3. Mahrholdt H, Wagner A, Judd RM, Sechtem U, Kim RJ (2005) Delayed enhancement cardiovascular magnetic resonance assessment of nonischaemic cardiomyopathies. European Heart Journal 26(15):14611474 .

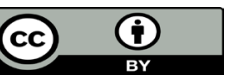

This work is licensed under Creative Commons Attribution 4.0 License

Submission Link: http://biomedres.us/submit-manuscript.php
4. Te Riele A, Tandri H, Bluemke DA (2014) Arrhythmogenic right ventricular cardiomyopathy (ARVC): cardiovascular magnetic resonance update. Journal of Cardiovascular Magnetic Resonance 16: 50.

5. Haugaa KH, Basso C, Badano LP, Bucciarelli Ducci C, Cardim N, et al. (2017) Comprehensive multi-modality imaging approach in arrhytmogenic cardiomyopathy- an expert consensus document of the European Association of Cardiovascular Imaging. European Heart Journal Cardiovascular Imaging 18(3):237-253.

6. Moss AJ, Hall WJ, Cannom DS, Daubert JP, Higgins SL, et al. (1996) Improved Survival with an Implanted Defibrillator in Patients with Coronary Disease at High Risk for Ventricular Arrhythmia. The New England Journal of Medicine 335(26):1933-1940.

7. Moss AJ, Greenberg H, Case RB, Zareba W, Hall WJ, et al. (2004) Longterm clinical course of patients after termination of ventricular tachyarrhythmia by an implanted defibrillator. Circulation 110(25): 3760-3765.

8. Camm J, Klein H, Nisam S (2007) The cost of implantable defibrillators: perceptions and reality. European Heart Journal 28(4): 392-397.

9. Zipes DP, Camm AJ, Borggrefe M, Buxton AE, Chaitman B, et al. (2006) ACC/AHA/ESC 2006 guidelines for management of patients with ventricular arrhythmias and the prevention of sudden cardiac death. Europace 8(9): 746-837.

10. Kober L, Thune JJ, Nielsen JC, Haarbo J, Videbaek L, et al. (2016) Defibrillator implantation in patients with nonischemic systolic heart failure. The New England Journal of Medicine 375(13): 1221-1230.

11. Pedretti S, Vargiu S, Baroni M, Dellegrottaglie S, Lanzarin B, et al. (2018) Complexity of Scar and Ventricular Arrhythmias in Dilated Cardiomyopathy of any Etiology: Long-Term Data from the SCARFEAR (cardiovascular magnetic Resonance predictors of Appropriate Implantable Cardioverter defibrillator therapy delivery Registry). Clinical Cardiology, in press.

12. Scott PA, Morgan JM, Carroll N, Murday DC, Roberts PR, et al. (2011) The Extent of Left Ventricular Scar Quantified by Late Gadolinium Enhancement MRI Is Associated With Spontaneous Ventricular Arrhythmias in Patients With Coronary Artery Disease and Implantable Cardioverter-Defibrillators. Circulation Arrhythmia \& Electrophysiology 4(3): 324-330.

13. Gulati A, Jabbour A, Ismail TF, Guha K, Khwaja J, et al. (2013) Association of Fibrosis With Mortality and Sudden Cardiac Death in Patients With Nonischemic Dilated Cardiomyopathy. JAMA 309(9): 896-908.

14. Halliday BP, Gulati A, Ali A, Guha K, Simon Newsome, et al. (2017) Association Between Midwall Late Gadolinium Enhancement and Sudden Cardiac Death in Patients With Dilated Cardiomyopathy and Mild and Moderate Left Ventricular Systolic Dysfunction. Circulation 135(22): 2106-2115. 